\title{
Frequency of Disc Degeneration at Different Levels of Lumbar Vertebrae in Adult Patients with Backache on Magnetic Resonance Imaging
}

\author{
Mishal Ijaz (Corresponding author)
}

Bachelors in Medical Imaging Technology, Student, Department of Allied Health Sciences, Superior College Lahore, University Campus, 17-KM Raiwaind Road, Kot Arain, Lahore, Pakistan

\section{Hajveriya Arshad}

Bachelors in Medical Imaging Technology, Student, Department of Allied Health Sciences, Superior College Lahore, University Campus, 17-KM Raiwaind Road, Kot Arain, Lahore, Pakistan

Iqra Shafi

Bachelors in Medical Imaging Technology, Student, Department of Allied Health Sciences, Superior College Lahore, University Campus, 17-KM Raiwaind Road, Kot Arain, Lahore, Pakistan

\section{Habiba Khalid}

Bachelors in Medical Imaging Technology, Student, Department of Allied Health Sciences, Superior College Lahore, University Campus, 17-KM Raiwaind Road, Kot Arain, Lahore, Pakistan

\section{Ahsan Ashraf}

Bachelors in Medical Imaging Technology, Student, Department of Allied Health Sciences, Superior College Lahore, University Campus, 17-KM Raiwaind Road, Kot Arain, Lahore, Pakistan

\section{Shafqat Rehman}

Masters in Medical Imaging Technology, Lecturer, Department of Allied Health Sciences, Superior College Lahore, University Campus, 17-KM Raiwaind Road, Kot Arain, Lahore, Pakistan

\section{Hafiz Muhammad Rizwan}

Masters in Medical Imaging Technology, Lecturer, Department of Allied Health Sciences, Superior College Lahore, University Campus, 17-KM Raiwaind Road, Kot Arain, Lahore, Pakistan

\author{
Rana Muhammad Athar Azeem Shams
}

Masters in Medical Imaging Technology, Lecturer, Department of Allied Health Sciences, Superior College Lahore, University Campus, 17-KM Raiwaind Road, Kot Arain, Lahore, Pakistan 
Masters in Mathematics, Lecturer, Department of Allied Health Sciences, Superior College Lahore, University Campus, 17-KM Raiwaind Road, Kot Arain, Lahore, Pakistan

\begin{abstract}
Objective:To Determine the Frequency of Disc Degeneration at Different Levels of lumbar Vertebrae in Adult Patients with lower back Pain on Magnetic Resonance Imaging.
\end{abstract}

Study Design: An analytical cross-sectional descriptive study was performed.

Settings: The study was performed in Bashir Neurospine Institute and Al-Razi Healthcare center, Lahore.

Period: An Analytical cross sectional study was conducted from 15 October,2020 to 15 January 2021.

Material and Methods: In our study, all those adult patients with lower back pain, obese, dull ache, numbness, tingling, pins and needle sensations, muscles spasms were included. And those patients who were not giving consent, spondylosis, lumbar spine stenosis, cervical and thoracic abnormalities, paedes, and those who were uncooperative were excluded. Data was tabulated and analyze with the help of statistical package for the social science (SPSS version 24). The data was reported using cross sectional descriptive statistics.

Results: In our research out of 160 patients 89 females and 51 males were those who diagnosed with disc degeneration and 10 females and 10 male in which disc degeneration was absent. 10 patients out of 160 patients were those who diagnosed with disc degeneration at the level of L1 - L2 between the age of 24 to 87, 8 patients were those in which disc degeneration present at the level of L2- L3 between the age of 43 to 87, 31 patients were those in which disc degeneration present at the level of L3-L4 and 96 patients diagnosed with disc degeneration present at the level of L4- L5 which is the lowest lumbar vertebra between the age of 55 to 80 years.

Overall out of 160 patients only 3 patients were those who represent with mild pain (25yrs) and 102 were those who came with moderate pain (27-90yrs) and 55 patients who represent with severe backache (30 to $89 \mathrm{yrs}$ ).

In this research out of $160(100 \%)$ patients only 102 patients $(63 \%)$ are those who present with moderate pain and 55 patients (34\%)with severe pain .At L1-L2 disc degeneration only 5 patients $(50 \%)$ were those who came with moderate and 5( 50\% )with severe pain. At L2-L3 disc degeneration two patients $(25 \%)$ with moderate and six patients (75\%)with severe pain .At L3-L4 disc degeneration 11 patients (35.4\%) with moderate and 20(64.5\%) with severe pain. At L4-L5 disc degeneration 51 patients (53\%) with moderate pain and 45 patients $(46.8 \%)$ with severe pain. Our study also revealed that elevated BMI patients had high frequency of disc degeneration.

Conclusion: This study conclude that lumbar disc degeneration was approximately in women than men who were represented with lower back pain. Minority of patients had normal lumbar MRI findings. Most of the degeneration occurs at the lowest lumbar vertebrae i.e. L4- L5 and patients who were presented with elevated BMI have increased risk of disc degeneration.

Keywords: Lumbar vertebrae, Magnetic resonance imaging, lower back pain. Degenerative disc disease.

DOI: $10.7176 / \mathrm{JHMN} / 90-17$

Publication date:June $30^{\text {th }} 2021$

\title{
1.Introduction
}

Degenerative disc disease (DDD) is a medical condition in which there are anatomic changes at different levels of vertebrae and a loss of function of one or more intervertebral discs of the spine. ${ }^{1}$ Disc disease simply refers to severe backache mainly due to wear and tear of spinal disc, causes radicular pain that radiates to legs. ${ }^{2}$ In America 2015 disc degeneration of whole spine was approximately in women than men with mean average of $77 \%$ and $71 \%$ respectively, mostly L4 and L5 level occurred almost $75.8 \%$ in women and $69.1 \%$ in men. ${ }^{3}$ prevalence of lower back pain among office workers of KEMU Lahore, Pakistan $29.20 \%$ and $69.20 \%{ }^{4}$ 
Between each vertebral body of the spine are pads of fibrocartilage based structure that provides support, flexibility and minor load shearing known as intervertebral disc. ${ }^{5}$ Unique structure of the spine allows flexion, extension, and rotation and bending. Intervertebral disc distribute the load of weight and work as a shock absorber. ${ }^{6}$

The number of people were increased who suffered from lower back pain and its related diseases. ${ }^{6}$ It is considerably a significant reduction in dehydration consequence is fall in the disc matrix proteoglycans and loss of water molecule which makes the disc stiff, compact and more susceptible to sprint in the exterior. ${ }^{7}$

Due to degeneration vertebral disc have inferior susceptibility for sustaining pressure, they bulge and loss heights. If single disc is degenerated, it can change structure and mechanism of lumbar spine.8Most commonly disc degeneration is occurred in young man than in woman and very noticeably in elderly women than in men, due to estrogen deficiency. Approximately $1 / 3$ rd of young females is affected by disc degeneration. ${ }^{9}$

Number of mechanical factors such as traumatic, genetics, aging and inflammation can highly affect the integrity of vertebral discs. ${ }^{10}$ Low back pain (LBP) is usually an expensive and problematic diagnostic and therapeutic challenge. Approximately $50-85 \%$ of adults are affected during their life time. ${ }^{11}$ In clinical practice MRI is most preferable modality for evaluation of vertebral disc degeneration. ${ }^{12}$ In T2 weighted image normal vertebral disc appear as hyper intense (the nucleus) which is surround by a dark ring (the annulus) although degenerative vertebral disc appears as dark, on T2 weighted images in which nucleus is not clearly differentiated from annulus. ${ }^{12}$ T1weighted sequences are typically used for morphological assessment of spinal structures. ${ }^{13}$ A hypointense signals on MRI does not means that it is symptomatic disc. ${ }^{14}$

Ravi raj Durganand Walwante et.al. (2017), researched on a descriptive cross-sectional Study on lumbar spine by using magnetic resonance imaging (MRI) with special recommendations to Modic changes and disc degeneration in rural area. A research study was conducted by Him on 165 patients; the age range was from 20 to 80 years whereby 82 of them were females. On lumbar Magnetic resonance imaging (MRI), overall prevalence of lumbar degenerative findings was $91 \%$. Disc degeneration is the most persistent finding seen in $91 \%$ patients and Modic changes in 38\%. Minority of participants ( $9 \%$ ) had normal lumbar MRI findings. Most of the degenerative findings were seen at lower lumbar levels i.e. L4/L5. ${ }^{15}$

Simo Sääksjärvi, et.al (2020), conducted a prospective follow up study disc degeneration of young low back pain patients. He conducted a research on 75 conscripts aged 20 years with LBP had their lumbar spine examined by MRI. At a follow-up of 30 years, the subjects were contacted; 35 of 69 filled a pain and disability questionnaire, and 26 of 35 were also reexamined clinically and by MRI. The images were evaluated for decreased SI and other degenerative changes. The total number of lumbar discs with decreased SI increased from 23 of 130 (18\%) to 92 of $130(71 \%$ - from 0.9 to 3.5 per subject during the follow-up. Distribution of DD changed from being mostly in L4-L5 and L5-S1 discs to being almost even between the four lowermost discs. Discs that had even slightly decreased SI at baseline were more likely to have severely decreased SI at follow-up, compared to healthy discs (57\% vs. $11 \%, \mathrm{P}<0.001)$. Other degenerative changes were also more common in these discs. Severity of DD at baseline did not have a significant association with current pain or disability. ${ }^{16}$

The aim of this study is to observe lumbar vertebral disc degeneration in adult symptomatic patients to provide data on frequency of lumbar disc degeneration at different levels of lumbar vertebrae. This categorized data will help medical participator's to correlate the backache with intervertebral disc issue and provide the higher authority to manage it at a society level.

\subsection{Material and Methods}

In our study, all those adult patients with lower back pain, obese, dull ache, numbness, tingling, pins and needle sensations, muscles spasms were included. And those patients who were not giving consent, spondylosis, lumbar spine stenosis, cervical and thoracic abnormalities, paedes, and those who were uncooperative were excluded. Data was tabulated and analyze with the help of statistical package for the social science (SPSS version 24). The data was reported using cross sectional descriptive statistics.

\section{Results}

In our research out of 160 patients 89 females and 51 males were those who diagnosed with disc degeneration and 10 females and 10 male in which disc degeneration was absent. 10 patients out of 160 patients were those who diagnosed with disc degeneration at the level of L1 - L2 between the age of 24 to 87, 8 patients were those in which disc degeneration present at the level of L2- L3 between the age of 43 to 87, 31 patients were those in which 
disc degeneration present at the level of L3-L4 and 96 patients diagnosed with disc degeneration present at the level of L4- L5 which is the lowest lumbar vertebra between the age of 55 to 80 years.

Overall out of 160 patients only 3 patients were those who represent with mild pain (25yrs) and 102 were those who came with moderate pain (27-90yrs) and 55 patients who represent with severe backache (30 to $89 \mathrm{yrs}$ ).

In this research out of $160(100 \%)$ patients only 102 patients $(63 \%)$ are those who present with moderate pain and 55 patients (34\%)with severe pain. At L1-L2 disc degeneration only 5 patients $(50 \%)$ were those who came with moderate and 5( 50\% )with severe pain. At L2-L3 disc degeneration two patients $(25 \%)$ with moderate and six patients (75\%)with severe pain .At L3-L4 disc degeneration 11 patients (35.4\%) with moderate and 20(64.5\%) with severe pain. At L4-L5 disc degeneration 51 patients $(53 \%)$ with moderate pain and 45 patients $(46.8 \%)$ with severe pain. Our study also revealed that elevated BMI patients had high frequency of disc degeneration.

\section{Discussion}

Our study was designed to determine the frequency of disc degeneration in adults who represented with complain of back pain on MRI. On the basis of diagnostic performance and sensitivity for the detection of even minor degenerative changes MRI is the most preferable modality for assessing patient having history of back pain. Current study data was collected according to variables like age,Gender, BMI ,level of vertebrae and level of pain. MRI was performed in those patients who present with different level of pain like (mild, moderate and severe).

In this research data of 160 patients were collected in which total number of males were 61 with $38.1 \%$ and female were 99 with $61.9 \%$ out of 160 patients 140 patients were those who diagnosed with lumbar disc degeneration $(87.5 \%$ )but in minority of patients 20 (12.5\%) lumbar disc degeneration was absent. Range of patient's age was between 20-91.

In another study Raviraj Durganand Walwante et.al. (2017), conducted a hospital based cross-sectional descriptive Study on lumbar spine by MRI with special reference to disc degeneration and Modic changes in rural area. He conducted a research on 165 patients; the age range was from 20 to 80 years whereby 82 of them were females. On MRI, overall prevalence of lumbar degenerative findings was 91\%. Disc degeneration was the most frequent finding seen in $91 \%$ patients and Modic changes in 38\%. Minority of participants (9\%) had normal lumbar MRI findings. The greater part of the degenerative findings were seen at lower lumbar levels for example L4/L5. ${ }^{15}$ And in our research out of 160 patients 89 females and 51 males were those who diagnosed with disc degeneration and 10 females and 10 male in which disc degeneration was absent . 10 patients out of 160 patients were those who diagnosed with disc degeneration at the level of L1 - L2 between the age of 24 to 87, 8 patients were those in which disc degeneration present at the level of L2- L3 between the age of 43 to 87, 31 patients were those in which disc degeneration present at the level of L3-L4 and 96 patients diagnosed with disc degeneration present at the level of L4- L5 which is the lowest lumbar vertebra between the age of 55 to 80 years.

Overall out of 160 patients only 3 patients were those who represent with mild pain (25yrs) and 102 were those who came with moderate pain (27-90yrs) and 55 patients who represent with severe backache (30 to $89 \mathrm{yrs}$ ).

In this research out of $160(100 \%)$ patients only 102 patients $(63 \%)$ are those who present with moderate pain and 55 patients (34\%)with severe pain .At L1-L2 disc degeneration only 5 patients $(50 \%)$ were those who came with moderate and 5( 50\% )with severe pain At L2-L3 disc degeneration two patients $(25 \%)$ with moderate and six patients (75\%)with severe pain .At L3-L4 disc degeneration 11 patients (35.4\%) with moderate and 20(64.5\%) with severe pain. At L4-L5 disc degeneration 51 patients (53\%) with moderate pain and 45 patients $(46.8 \%)$ with severe pain. Our study also revealed that elevated BMI patients had high frequency of disc degeneration.

\section{Conclusion}

This study conclude that lumbar disc degeneration was approximately in women than men who were represented with lower back pain. Minority of patients had normal lumbar MRI findings. Most of the degeneration occurs at the lowest lumbar vertebrae i.e. L4- L5 and patients who were presented with elevated BMI had increased risk of disc degeneration.

\section{Conflict if interest}

There is no conflict of interest in this research. 


\section{References}

1. Fardon DF, Williams AL, Dohring EJ, Murtagh FR, Rothman SL, Sze GK. Lumbar disc nomenclature: version 2.0: Recommendations of the combined task forces of the North American Spine Society, the American Society of Spine Radiology and the American Society of Neuroradiology. The Spine Journal. 2014 Nov 1;14(11):2525-45..

2. Hayes AJ, Melrose J. Electro-Stimulation, a Promising Therapeutic Treatment Modality for Tissue Repair: Emerging Roles of Sulfated Glycosaminoglycans as Electro-Regulatory Mediators of Intrinsic Repair Processes. Advanced Therapeutics.:2000151.

3. Brinjikji W, Luetmer PH, Comstock B, Bresnahan BW, Chen LE, Deyo RA, Halabi S, Turner JA, Avins AL, James K, Wald JT. Systematic literature review of imaging features of spinal degeneration in asymptomatic populations. American Journal of Neuroradiology. 2015 Apr 1;36(4):811-6.

4. Arslan, S.A., Hadian, M.R., Olyaei, G., Bagheri, H., Yekaninejad, M.S., Ijaz, S. and Kheradmand, A.A., 2016. Prevalence and risk factors of low back pain among the office workers of King Edward Medical University Lahore, Pakistan. Physical Treatments-Specific Physical Therapy Journal, 6(3), pp.161-168.

5. Donnally III, Chester J., Andrew Hanna, and Matthew Varacallo. "Lumbar degenerative disk disease." (2019).

6. Kim, H.S., Wu, P.H. and Jang, I.T., 2020. Lumbar degenerative disease part 1: Anatomy and pathophysiology of intervertebral discogenic pain and radiofrequency ablation of basivertebral and sinuvertebral nerve treatment for chronic discogenic back pain: A prospective case series and review of literature. International journal of molecular sciences, 21(4), p.1483.

7. Shankar H, Scarlett JA, Abram SE. Anatomy and pathophysiology of intervertebral disc disease. Techniques in Regional Anesthesia and Pain Management. 2009 Apr 1;13(2):67-75.

8. Colombini A, Lombardi G, Corsi MM, Banfi G. Pathophysiology of the human intervertebral disc. The international journal of biochemistry \& cell biology. 2008 Jan 1;40(5):837-42.

9. Schwarz-Nemec U, Friedrich KM, Prayer D, Trattnig S, Schwarz FK, Weber M, Bettelheim D, Grohs JG, Nemec SF. Lumbar intervertebral disc degeneration as a common incidental finding in young pregnant women as observed on prenatal magnetic resonance imaging. Journal of Women's Health. 2020 May 1;29(5):713-20.

10. Molinos M, Almeida CR, Caldeira J, Cunha C, Gonçalves RM, Barbosa MA. Inflammation in intervertebral disc degeneration and regeneration. Journal of the Royal Society Interface. 2015 Mar 6;12(104):20141191.

11. Yang H, Haldeman S, Lu ML, Baker D. Low back pain prevalence and related workplace psychosocial risk factors: a study using data from the 2010 National Health Interview Survey. Journal of manipulative and physiological therapeutics. 2016 Sep 1;39(7):459-72.;

12. Duarte RM, Vaccaro AR. Spinal infection: state of the art and management algorithm. European Spine Journal. 2013 Dec 1;22(12):2787-99.

13. Zanchi F, Richard R, Hussami M, Monier A, Knebel JF, Omoumi P. MRI of non-specific low back pain and/or lumbar radiculopathy: do we need T1 when using a sagittal T2-weighted Dixon sequence? European radiology. $2020 \mathrm{Feb}$ 4:1-1.

14. . Kim HS, Wu PH, Jang IT. Lumbar degenerative disease part 1: Anatomy and pathophysiology of intervertebral discogenic pain and radiofrequency ablation of basivertebral and sinuvertebral nerve treatment for chronic discogenic back pain: A prospective case series and review of literature. International journal of molecular sciences. $2020 \mathrm{Jan} ; 21(4): 1483$.

15. Walwante R, Dhapate S, Porwal S. Study of lumbar spine by MRI with special reference to disc degeneration and Modic changes in rural area. Indian Journal of Clinical Anatomy and Physiology. 2017 Oct;4(4):569-73.

16. Sääksjärvi S, Kerttula L, Luoma K, Paajanen H, Waris E. Disc Degeneration of Young Low Back Pain Patients: A Prospective 30-year Follow-up MRI Study. Spine. 2020 Oct 1;45(19):1341-7. 


\section{Cross Tabulation}

\begin{tabular}{llllll} 
Gender & & & & & \\
& & Frequency & Percent & Valid Percent & Cumulative Percent \\
\hline Valid & Female & 99 & 61.9 & 61.9 & 61.9 \\
\cline { 2 - 6 } & Male & 61 & 38.1 & 38.1 & 100.0 \\
\cline { 2 - 6 } & Total & 160 & 100.0 & 100.0 & \\
\hline
\end{tabular}

\begin{tabular}{|c|c|c|c|c|c|}
\hline & & & Degenera & & \\
\hline & & Frequency & Percent & Valid Percent & $\begin{array}{c}\text { Cumulative } \\
\text { Percent }\end{array}$ \\
\hline Valid & $\mathrm{NO}$ & 20 & 12.5 & 12.5 & 12.5 \\
\hline & YES & 140 & 87.5 & 87.5 & 100.0 \\
\hline & Total & 160 & 100.0 & 100.0 & \\
\hline
\end{tabular}

\begin{tabular}{|c|c|c|c|c|}
\hline \multirow{3}{*}{ Count } & \multicolumn{4}{|c|}{ Gender * Disc Degeneration Cross tabulation } \\
\hline & & \multicolumn{2}{|c|}{ Disc Degeneration } & \multirow[b]{2}{*}{ Total } \\
\hline & & $\mathrm{NO}$ & YES & \\
\hline \multirow[t]{2}{*}{ Gender } & Female & 10 & 89 & 99 \\
\hline & Male & 10 & 51 & 61 \\
\hline Total & & 20 & 140 & 160 \\
\hline
\end{tabular}

\section{Graph 1:}

Gender vs. Disc Degeneration

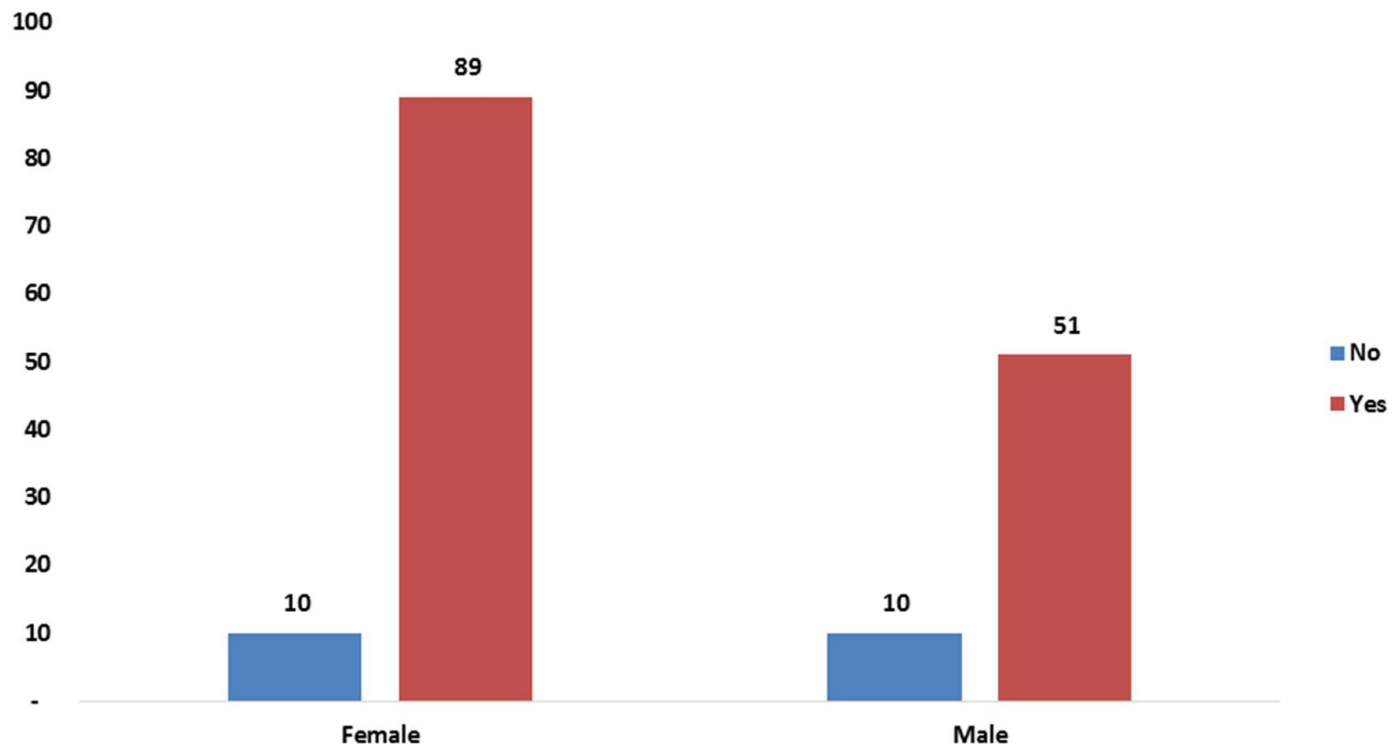


Graph 2:

\section{Age Group vs. Level of Pain}

25

20

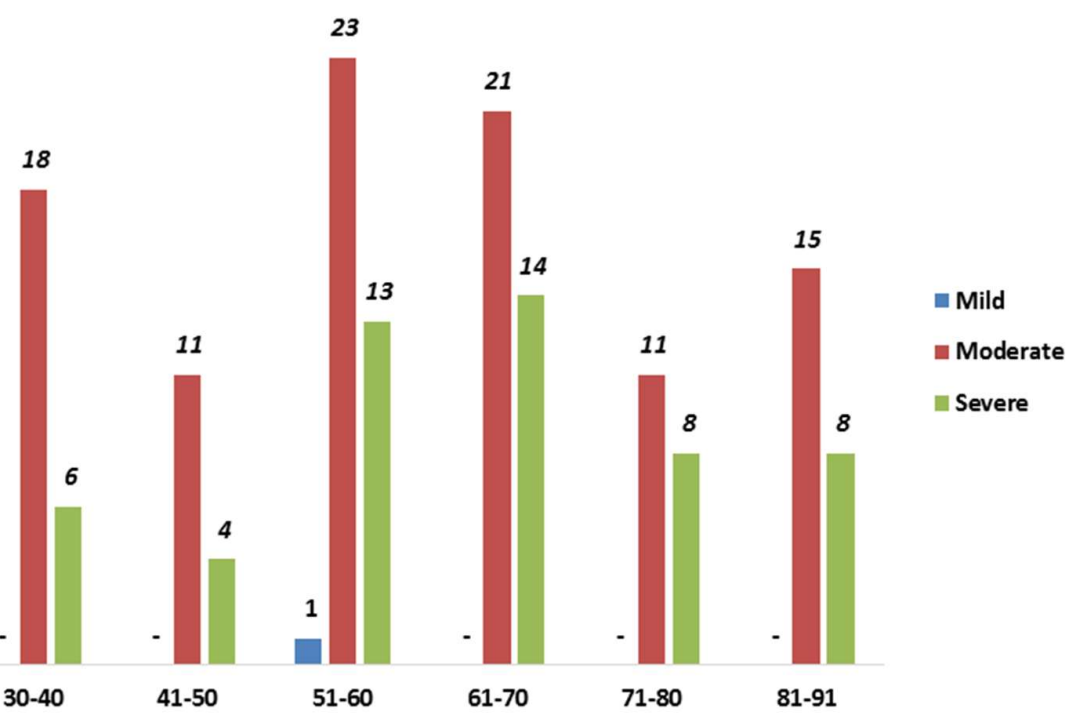

